\title{
Implementation of HACCP management system: Case study of a baking industry (cake) in Dhaka, Bangladesh.
}

\author{
${ }^{1}$ Md. Fahad Jubayer ${ }^{\mathrm{a}, *},{ }^{1}$ Md. Sajjad Hossain ${ }^{\mathrm{b}}$, Md. Al-Emran ${ }^{\mathrm{c}}$, Md. Nasir Uddin ${ }^{\mathrm{c}}$ \\ ${ }^{a}$ Department of Food Engineering \& Technology, Sylhet Agricultural University, Sylhet- \\ 3100, Bangladesh. \\ ${ }^{b}$ Department of Chemical and Food Process Engineering, Rajshahi University of \\ Engineering and Technology, Rajshahi, Bangladesh. \\ ${ }^{c}$ Department of Food Technology and Rural Industries, Bangladesh Agricultural University, \\ Mymensingh-2202, Bangladesh.
}

\section{${ }^{1}$ Equal contribution}

*Corresponding author:

Md. Fahad Jubayer, Department of Food Engineering \& Technology, Sylhet Agricultural University, Sylhet-3100, Bangladesh.

Email: fahadbau21@hotmail.com

Phone: +88-01876-529642.

\begin{abstract}
The study aims to provide technical information on the development and application of hazard analysis and critical control points (HACCP) in one of Dhaka's popular baking (cake) industries. A generic HACCP plan in accordance with legal requirements was created after a detailed analysis of data collected from the company. Every step of the production was examined for biological, chemical, and physical hazards. The prerequisite program was designed to address some hazards prior to production, thereby simplifying the HACCP plan. The critical control points were determined by answering the questions in the decision trees. Finally, the HACCP control chart was created to include critical limits, monitoring, and corrective action as components of several HACCP principles. One critical control point (CCP) and two operational pre-requisite programs (oPRPs) were identified throughout the manufacturing process.
\end{abstract}

Keywords: HACCP; baking; food safety; critical control points; prerequisite program; hazard analysis. 


\section{Abbreviations}

HACCP, hazard analysis and critical control point; GHP, Good hygiene practice; GMP, Good manufacturing practice; BABBMA, Bangladesh Auto Biscuit and Bread Manufacturers' Association; CCP, critical control point; FSMS, Food Safety Management System; PRPs, prerequisites programs; oPRPs, operational prerequisites programs; BDS, Bangladesh standards; CL, critical limit; HA, hazard analysis; HRM, human resource management; RI, Risk index; CoA, Certificate of assurance; QA, Quality assurance; FIFO, First in first out; SOP, Standard operating procedure; MC, Moisture content; $\mathrm{a}_{\mathrm{w}}$, water activity; PLC, Programmable logic control.

\section{Introduction}

Cakes are popular sweet bakery products all over the world. Globally, the cake market is expected to grow at a rate of $3.3 \%$ per year from 2018 to 2023, reaching a value of $\$ 75$ billion by 2023 (Konstantas et al., 2019, Mordor Inteligence, 2018). Cakes are available in an enormous variety of forms and recipes, making them popular among people of all ages and demographics around the world. The primary raw materials of cake include flour, sugar, egg, shortening (Matsakidou et al., 2010), color, flavor, salt, and emulsifier, which are then processed into finished goods via various processing steps such as mixing, baking, cooling, and packaging (Conforti, 2006). Food-borne illnesses are usually associated with this kind of processed products. Escherichia coli $\mathrm{O} 157: \mathrm{H} 7$ has been found in cheese and pizza, Listeria monocytogenes in cheese and ice cream, and Salmonella typhimurium in peanut butter (Centers for Disease Control and Prevention, 2020). Between 2004 and 2013, there were 142 cases of food-borne disease outbreaks and 2822 illnesses associated with bakery products in the United States (CSPI, 2015). Numerous food safety hazards associated with cake raw materials have been discovered, including Aflatoxin B1 (Riba et al., 2010), mycotoxins (Liu et al., 2015), E. coli, Salmonella spp., Bacillus cereus, and several pathogenic microorganisms in flour (Wu et al., 2017). Besides, presence of Salmonella (Foley et al., 2013), Campylobacter (Ricke et al., 2013b), Listeria (Jones et al., 2012) are very common in the raw eggshell. Various types of hazards can be introduced during the cake manufacturing process through different processing steps such as food raw materials, processing equipment, processing environment, and food handlers.

The food industry is responsible not only for producing safe foods, but also for demonstrating how food safety issues are designed and implemented. Developing a food 
safety management system contributes to this goal in an open and transparent manner (Shuvo et al., 2019). The HACCP system is a powerful and efficient tool for producing safe food. In the food production system, the HACCP tool application is a systematic science-based system that specifies specific food safety hazards. It determines the food safety control measure (FAO \& WHO., 2003). Every HACCP system is capable of addressing and accommodating changes in technological advancement, advancement in equipment design, adjustments in processing procedures, and so on. Principles of the HACCP system have been adopted by the Codex Alimentarius Commission (CAC), and application guidelines are presented in an Annex to the General principles of food hygiene (FAO and WHO, 2003). HACCP implementation is based on seven principles that are used to identify and control hazards to an acceptable level. Before implementing a HACCP plan, a strong prerequisite program should be in place. Prerequisite programs (for example, sanitation, pest control, personnel practices, GHP, ingredient and product specifications, staff training, cleaning and disinfectant regimes, and hygienically designed facilities) are used to ensure that employees, equipment, premises, and transportation do not contribute to or become food safety hazards (FAO \& WHO. 2003, Walker et al., 2003). Subsequently, HACCP is a globally recognized, effective, and preventive food safety management system for measuring hazards, estimating risks, and establishing precise control measures that emphasize prevention and control rather than reliance on end-product testing and traditional inspection methods. Implementing HACCP is more effective than traditional quality assurance methods in gaining consumer trust and establishing a good corporate image (Shuvo et al., 2019).

Food contamination and adulteration have recently emerged as major public health concerns in Bangladesh. As a result, an independent food safety body named BFSA was formed in 2014. According to the BABBMA, there are approximately 60 large factories registered in Bangladesh that manufacture bakery products (BAPA, 2021). Pesticide residues and postprocessing contamination with pathogenic microbes such as Salmonella and E. coli are the most prevalent food safety risks in this sector (Suman et al., 2021). Bangladesh has a moderate level of food safety inspection, though the leading private food industries have the internal capacity to ensure food safety compliance with US and European standards (Suman et al., 2021). This current study aimed to implement the HACCP system in the cake manufacturing plant in order to identify CCPs, thereby establishing an effective preventive system that will lead to safer and more efficient cake production. The application of the HACCP system can effectively ensure the quality and safety of the final product. 


\section{Materials and Methods}

\subsection{Company description}

This study was conducted in a baking industry located in the Dhaka division of Bangladesh, which was a joint venture manufacturing. The research was carried out from January to August of 2019. It was classified as a medium-scale plant, with a monthly production capacity of 500 tons and a workforce of around 200 people during the study period. Muffins, plain cakes, fruit cakes, Swiss rolls, and sponge cakes were among the products produced by the industry. In addition to the domestic market, the products had begun to be exported directly to international markets. The restructuring was intended to expand the company's market. As a result, the company planned to implement an effective FSMS to ensure consumer food safety and high-quality products. This study explains the PRPs, OPRPs, and HACCP principles in accordance with the standard requirements for the cake manufacturing line. The company layout shown below is adopted from the corresponding authors' previous work (Jubayer et al., 2020). He has conducted additional research in the same industry in 2020.

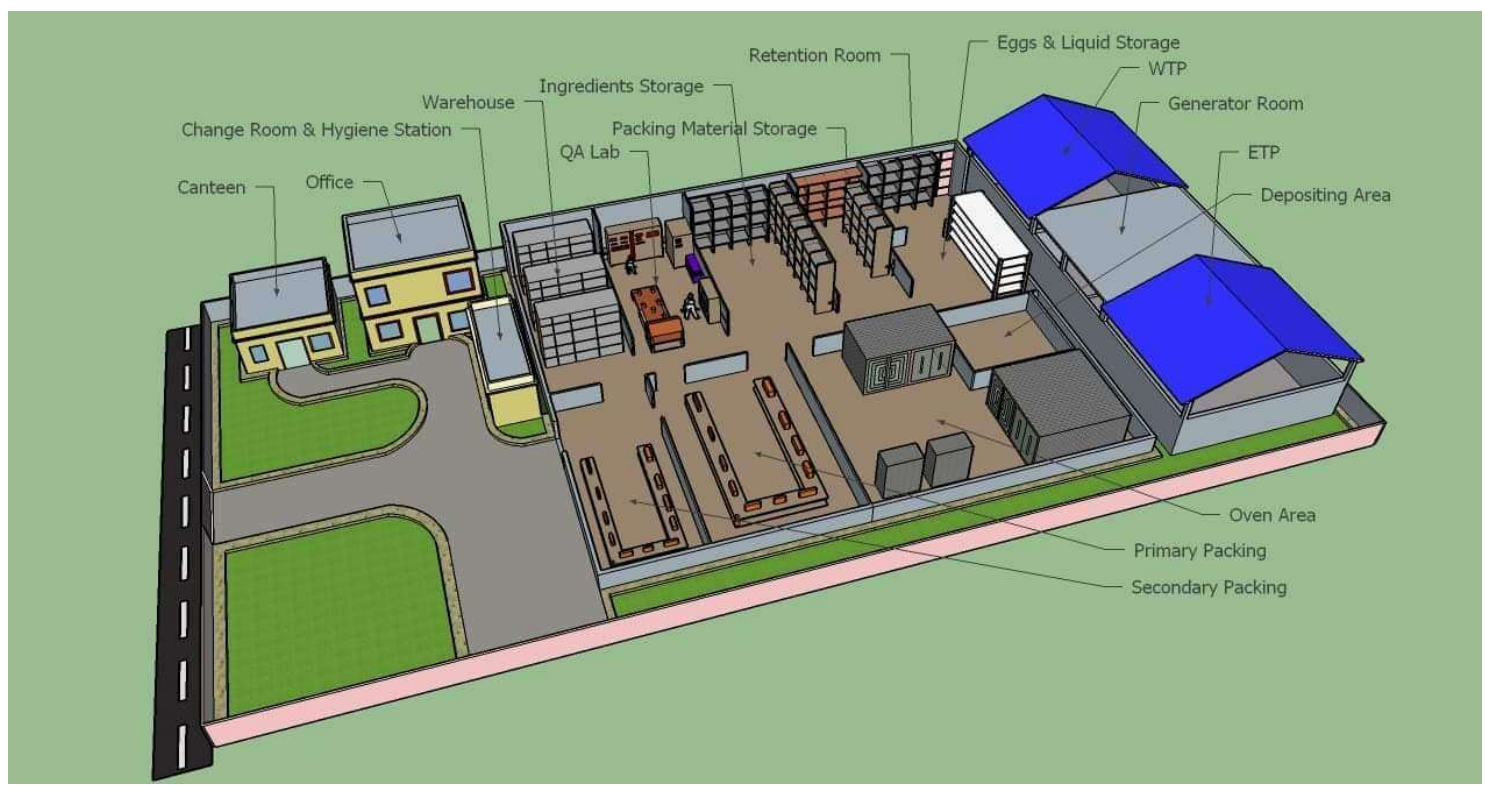

Figure 1. Layout of the plant (Jubayer et al., 2020)

\subsection{HACCP implementation steps}

HACCP is a scientific and systematic approach to food safety that identifies and controls specific food safety hazards. Implementing HACCP is a symbol of product quality and safety 
that ensures consumer satisfaction. According to Codex Alimentarius, the HACCP system is based on seven scientific principles (CAC, 1997).

Principle 1: Perform hazard analysis (HA) for the identification of physical, chemical, and biological hazards.

Principle 2: Identification of critical control points (CCPs) according to the decision tree.

Principle 3: Establishing critical limits (CL) to ensure uniformity in the safety analyses of the processes involved.

Principle 4: Monitoring each CCP.

Principle 5: Establishing corrective actions to be executed when monitoring indicates a deviation from a predefined critical limit.

Principle 6: Establishing effective record-keeping procedures.

Principle 7: Establishing procedures for verification that the HACCP system is working correctly.

In this study PRPs and OPRPs are applied along with HACCP plan throughout the whole production process.

\section{Results and discussion}

\subsection{Implementation of HACCP plan}

\subsubsection{Listing the prerequisite (PRP)}

Prior to the HACCP system, PRPs are systematically applied in the food processing sector to support and improve the system's effectiveness and efficiency. PRP application is considered as the foundation of HACCP system. PRP represents the formalization of GMP and GHP elements (Wallace \& Williams, 2001). The PRPs were: PRP environmental hygiene, PRP establishment- design and facilities, PRP premises and rooms, PRP equipment, PRP water supply, PRP drainage and waste disposal, PRP cleaning \& sanitation, PRP personnel hygiene facilities and toilets, PRP temperature and air quality control, PRP lighting, PRP handling, storage \& transportation, PRP control of operation, PRP pest control, PRP waste management, PRP traceability, and PRP training. 


\subsubsection{Preliminary steps to enable hazard analysis}

\subsubsection{The HACCP team}

A team was formed to implement an efficient HACCP system. While assembling the HACCP team for the baking industry, the following factors were taken into account. The team was supposed to have six members.

a) In terms of qualifications and experience, the members must be competent in their particular fields of activity.

b) The team should be led by a team leader who will direct the activities.

c) The team coordinator will be in charge of developing, implementing, and maintaining the HACCP system.

d) All team members are expected to receive proper training.

e) The team must develop and maintain all documents in accordance with the HACCP system.

f) Furthermore, if there is a change or modification in the process, the team must examine the adequacy of the HACCP system on a yearly basis.

Thus the formation of the HACCP team was as follows.

HACCP team leader

HACCP team coordinator

HACCP team members
Plant manager

Manufacturing manager

Quality Assurance Manager

Manager- HRM \& Compliance

Ware House Manager

Maintenance Manager (Electrical)

Maintenance Manager (Mechanical)

Supply Chain Manager

The team leader, as the highest authority, maintains the smooth operation of the industry and ensures that all legal criteria for its proper operation are met. It is also his obligation to supervise all phases of the plan, as well as the responsibilities and coordination of all internal and external corporate operations (Pombo Marques et al., 2012). The team members are responsible for keeping and regulating the records related to their area of operation. 


\subsubsection{Product characteristics and intended use}

The HACCP team needs to start with a thorough description of the produces, defining their composition, chemical, biological, and physical properties. Table 1 shows the generic framework for the description of cake.

Table 1. Product description and intended use of cakes

\begin{tabular}{|c|c|c|}
\hline Product & \multicolumn{2}{|l|}{ Cake is a bakery product } \\
\hline Product composition & \multicolumn{2}{|c|}{$\begin{array}{l}\text { Wheat flour, sugar, eggs, salt, vegetable fat, glycerol, starch, wheat } \\
\text { starch, skim milk powder, raising agents (E 500), preservative (E 202), } \\
\text { and flavor. }\end{array}$} \\
\hline \multirow[t]{17}{*}{ Product characteristics } & Physico-chemical characteristics: & \\
\hline & Moisture & $18-22 \% \mathrm{wb}$ \\
\hline & Water activity $\left(\mathrm{a}_{\mathrm{w}}\right)$ & $0.76-0.81$ \\
\hline & $\begin{array}{l}\text { Acid Value of extracted fat, (as oleic acid), } \\
\text { percent by mass, Max. }\end{array}$ & 1.0 \\
\hline & Microbiological characteristics: & \\
\hline & Total viable count, $\mathrm{cfu} / \mathrm{g}$, Max. & 20000 \\
\hline & Enterobacteriaceae spp. & $<10^{2} \mathrm{UFC} / \mathrm{g}$ \\
\hline & Staphylococcus aureus & $<10^{2} \mathrm{UFC} / \mathrm{g}$ \\
\hline & Salmonella spp. & Absent \\
\hline & Listeria monocytogenes & Absent \\
\hline & Coliform & Absent \\
\hline & Mold cfu/g, maximun & 50 \\
\hline & Sensory characteristics: & \\
\hline & Physical condition & Solid \\
\hline & Color & Characteristics \\
\hline & Flavor & Typical \\
\hline & Texture & Typical \\
\hline Labeling requirements & \multicolumn{2}{|c|}{$\begin{array}{l}\text { Product's name, composition, allergen information, origin, } \\
\text { manufactures date, expiry date, destination, net weight, storage } \\
\text { temperature, and destination. }\end{array}$} \\
\hline $\begin{array}{l}\text { Storage and transport } \\
\text { Conditions }\end{array}$ & \multicolumn{2}{|c|}{$\begin{array}{l}\text { Primary packages should be packaged further in a secondary packing } \\
\text { procedure (gift boxes). Secondary boxed must packed in tertiary } \\
\text { packaging (cartons). Final storage and transportation should make at } \\
\text { ambient temperature. }\end{array}$} \\
\hline
\end{tabular}




\begin{tabular}{|l|l|}
\hline Shelf life & 4 months \\
\hline Intended use & $\begin{array}{l}\text { Direct consumption. The product mentioned is for the general } \\
\text { population, except for gluten sensitive groups }\end{array}$ \\
\hline Applicable laws & $\begin{array}{l}\text { BDS 1574, specifications for cakes; BDS 1240, specifications for } \\
\text { drinking water; BDS 381, specifications for maida; BDS 1567, } \\
\text { specifications for refined oil; BDS 79, specifications for jams and } \\
\text { jellies; BDS 1615, specifications for cocoa powder; BDS 138, } \\
\text { specifications for refined sugar; BDS 207, specifications for milk } \\
\text { powder and cream powder; BDS 822, Code of hygienic conditions for } \\
\text { food processing units etc. }\end{array}$ \\
\hline
\end{tabular}

\subsubsection{Construction of flow diagram}

A flowchart showing the whole manufacturing process of cake was constructed (Figure 1). The HACCP team checked the flow diagrams on-site. Only a well-planned, informative, and well analyzed flowchart helps to facilitate overall production process, making it much easier to detect potential deviations (Pombo Marques et al., 2012). 


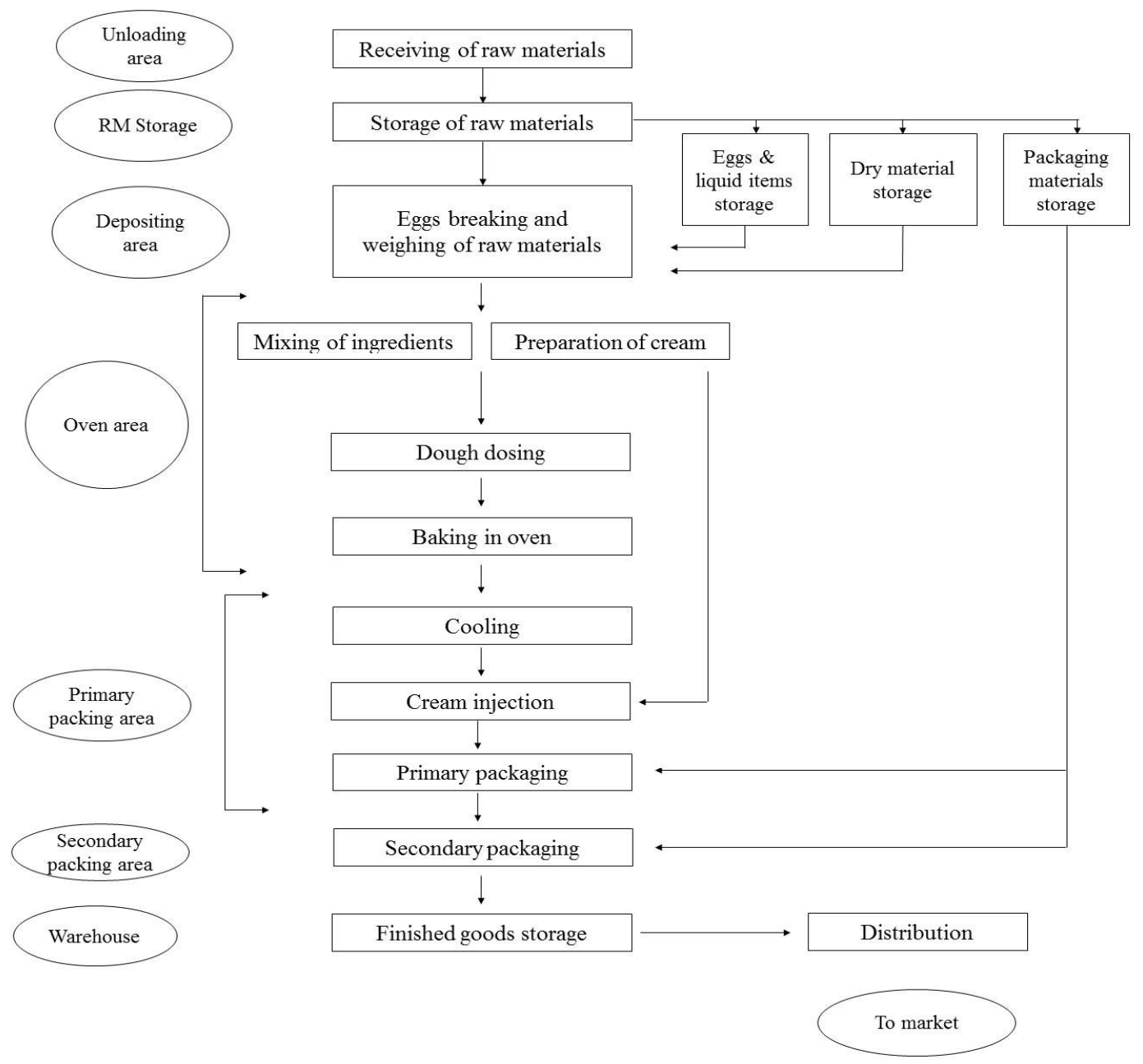

Figure 1. Complete flowchart of the cake manufacturing process.

\subsubsection{Hazard analysis, identification of CCP's and oPRPs}

The hazard analysis assists in identifying potential hazards associated with the entire process, from raw material receipt to product delivery to consumers. During the hazard analysis, hazards were further categorized into three categories: a) biological, b) physical, c) chemical, and (Fernández-Segovia et al., 2014). Hazard analysis or identification is generally aimed to identify potential threats to human health that may be introduced into baked goods during the manufacturing process. Based on the hazard analysis, further risk assessments were 
accomplished. In the process of risk assessment, customer complaints, shipment returns, and laboratory test results were typically taken into account (Batista et al., 2003). Hazards as identified for each step of the process flow diagram were entered in the format. Following the completion of the subsequent process steps, values for the likelihood of the hazard's presence and the consequences of the hazards were entered into the assessment column. The values were determined in the following way (Table 2 and Table 3).

Table 2. Likelihood and consequence criteria to specify potential hazard in cake manufacturing process

\begin{tabular}{|l|l|}
\hline \multicolumn{1}{|c|}{ Likelihood ( Probability ) } & \multicolumn{1}{c|}{ Consequences ( Severity ) } \\
\hline 4. Frequent - e.g. Daily & 4. Very High ( Catastrophe ) - e.g. Death \\
\hline 3. Likely - e.g. Weekly & 3. High ( Critical ) - e.g. Illness \\
\hline 2. Occasional -e.g. Monthly & 2. Medium - e.g. Injury \\
\hline 1. Unlikely - e.g. Yearly & 1. Minor - e.g. No Injuries \\
\hline
\end{tabular}

The likelihood, i.e. probability, and consequences of a specified hazardous event occurring are combined to form risk. Thus it can be defined as,

\section{Risk index $(\mathrm{RI})=$ Likelihood $\times$ Consequences}

Significant hazards were identified using a numerical scale of one (1) to four (4) of likelihood and consequence. When rating likelihood and consequences, previous experiences, records, and data were considered (Table 3).

Table 3. Analysis of likelihood $v s$ consequences

\begin{tabular}{|l|c|c|c|}
\hline \multicolumn{1}{|c|}{ Hazard types } & $\begin{array}{c}\text { Likelihood } \\
\text { (Probability) }\end{array}$ & $\begin{array}{c}\text { Consequences } \\
\text { (Severity) }\end{array}$ & RI \\
\hline Physical: (Stones, Sand, Husks, and Plastic) & $\mathrm{x}$ & $\mathrm{y}$ & $\mathrm{Xy}$ \\
\hline $\begin{array}{l}\text { Chemical: (Mercury (Hg), Chromium (Cr), } \\
\text { Aflatoxin, Melamine) }\end{array}$ & $\mathrm{x}$ & $\mathrm{y}$ & $\mathrm{Xy}$ \\
\hline $\begin{array}{l}\text { Biological: Total Plate Count (TPC), Coli } \\
\text { form, Molds and Pests }\end{array}$ & $\mathrm{x}$ & $\mathrm{y}$ & $\mathrm{Xy}$ \\
\hline
\end{tabular}


According to Table 3, the IR for this current study varies between 1 and 12. The calculated risk index shows that, if the RI is higher than 8 , then it should be managed by the HACCP plan. HACCP plan is a document prepared in accordance with the seven principles of HACCP (Pombo Marques et al., 2012). On the other hand, when the IR is less than 4, the results should be maintained by the oPRPs (Pombo Marques et al., 2012). The RI for the production of cake is shown in Table 4.

Table 4. Risk index and its management (Pombo Marques et al., 2012)

\begin{tabular}{|c|c|c|}
\hline RI & Risk type & Management of risk \\
\hline $\mathrm{RI} \leq 4$ & Satisfactory risk & $\begin{array}{c}\text { Should be managed by } \\
\text { oPRPs }\end{array}$ \\
\hline $4 \geq \mathrm{RI} \leq 8$ & Lower risk & \multirow{2}{*}{$\begin{array}{c}\text { Should be managed by the } \\
\text { HACCP plan }\end{array}$} \\
\hline $12 \geq \mathrm{RI} \leq 16$ & Increased risk & Critical risk \\
\hline
\end{tabular}

After completing the hazard assessment, relative control measures are selected with the help of the CCP decision tree Figure 2. CCP is a step in which a specific control measure must be implemented in order to prevent or eliminate a food safety hazard or reduce the risk to an acceptable level (ISO 22000, Section 3.10). 


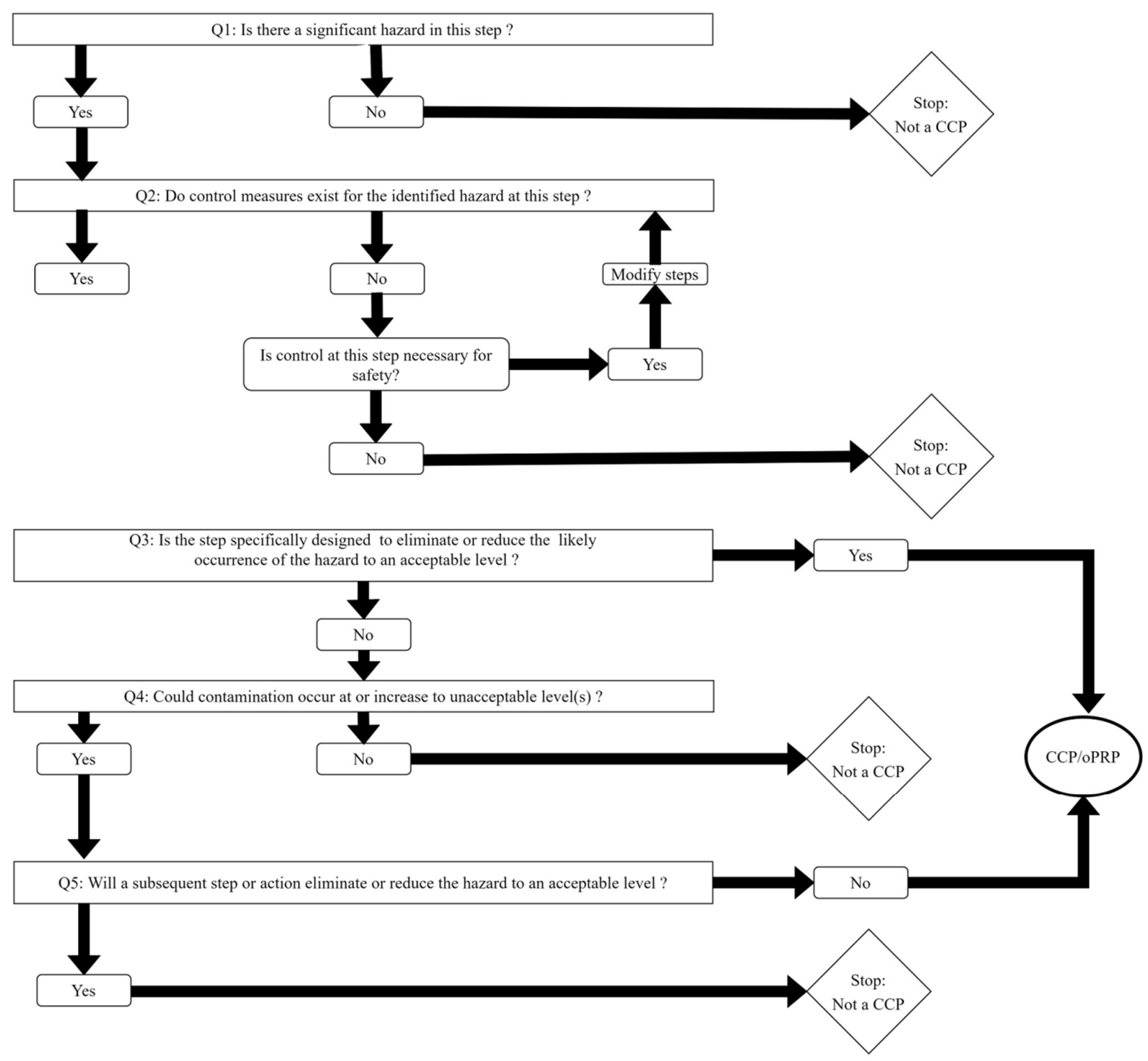

Figure 2. CCP decision tree for HACCP implementation (adopted from Allata et al., 2017)

An oPRP's components are the same as those of a CCP, with the exception that no critical limit is required for control measure(s) (Allata et al., 2017) and failure in the manufacturing process has no direct effect on the process (Pombo Marques et al., 2012). The hazard analysis of the cake manufacturing process is shown at Table 5 .

Table 5. Hazard analysis for the production process of cake

\begin{tabular}{|c|c|c|c|c|c|c|c|c|c|c|c|c|c|}
\hline \multirow{2}{*}{$\begin{array}{c}\text { Processing } \\
\text { step }\end{array}$} & \multirow[t]{2}{*}{ Hazards } & \multirow[t]{2}{*}{ Risk effects } & \multirow[t]{2}{*}{ Probable causes } & \multicolumn{3}{|c|}{ Risk evaluation } & \multirow{2}{*}{$\begin{array}{c}\text { Control Measures } \\
\text { ( SOP's or Work } \\
\text { Instructions) }\end{array}$} & \multicolumn{5}{|c|}{ Decision tree } & \multirow[t]{2}{*}{ Record } \\
\hline & & & & $\mathrm{P}$ & $\mathrm{S}$ & RI & & Q1 & Q2 & Q3 & Q4 & Q5 & \\
\hline
\end{tabular}




\begin{tabular}{|c|c|c|c|c|c|c|c|c|c|c|c|c|c|}
\hline \multirow[t]{3}{*}{$\begin{array}{l}\text { Receiving } \\
\text { of raw and } \\
\text { packaging } \\
\text { materials }\end{array}$} & $\begin{array}{l}\text { Physical: Foreign } \\
\text { objects, such as } \\
\text { hair, insects, broken } \\
\text { plastics etc. }\end{array}$ & $\begin{array}{l}\text { Without } \\
\text { consequences }\end{array}$ & $\begin{array}{l}\text { Packaging, } \\
\text { hygiene, pest } \\
\text { control. }\end{array}$ & 3 & 1 & 3 & Visual inspection & $\mathrm{Y}$ & $\mathrm{Y}$ & $\mathrm{N}$ & $\mathrm{Y}$ & $\mathrm{Y}$ & PRP \\
\hline & $\begin{array}{l}\text { Chemical: Acidity, } \\
\text { melamine, heavy } \\
\text { metals, \& residual } \\
\text { chemical etc. }\end{array}$ & $\begin{array}{l}\text { Without } \\
\text { consequences }\end{array}$ & CoA, packaging. & 1 & 3 & 3 & $\begin{array}{l}\text { Supplier evaluation, CoA, } \\
\text { HACCP certificate, } \\
\text { personal and transport } \\
\text { hygiene status, lab test } \\
\text { (QA) }\end{array}$ & $\mathrm{Y}$ & $\mathrm{Y}$ & $\mathrm{N}$ & $\mathrm{Y}$ & $\mathrm{Y}$ & PRP \\
\hline & $\begin{array}{l}\text { Biological: } \\
\text { Microbial } \\
\text { contamination } \\
\text { (pathogenic). }\end{array}$ & $\begin{array}{l}\text { With } \\
\text { consequences }\end{array}$ & CoA, hygiene. & 1 & 4 & 4 & $\begin{array}{l}\text { Supplier evaluation, CoA, } \\
\text { HACCP certificate, } \\
\text { personal and transport } \\
\text { hygiene status, lab test } \\
\text { (QA) }\end{array}$ & $\mathrm{Y}$ & $\mathrm{Y}$ & $\mathrm{N}$ & $\mathrm{Y}$ & $\mathrm{Y}$ & PRP \\
\hline \multirow[t]{3}{*}{$\begin{array}{l}\text { Storage of } \\
\text { raw and } \\
\text { packaging } \\
\text { materials }\end{array}$} & $\begin{array}{l}\text { Physical: Foreign } \\
\text { objects, insects. }\end{array}$ & $\begin{array}{l}\text { Without } \\
\text { consequences }\end{array}$ & $\begin{array}{l}\text { Irregular } \\
\text { inspection and } \\
\text { improper } \\
\text { hygiene. }\end{array}$ & 3 & 1 & 3 & $\begin{array}{l}\text { Keep storage } \\
\text { environment safe, keep } \\
\text { containers closed. } \\
\text { Separate areas for raw } \\
\text { and packaging materials. }\end{array}$ & $\mathrm{Y}$ & $\mathrm{Y}$ & $\mathrm{N}$ & $\mathrm{N}$ & & PRP \\
\hline & $\begin{array}{l}\text { Chemical: } \\
\text { Production of } \mathrm{H}_{2} \mathrm{~S} \\
\text { (eggs) }\end{array}$ & $\begin{array}{l}\text { Without } \\
\text { consequences }\end{array}$ & $\begin{array}{l}\text { Storage } \\
\text { temperature }\end{array}$ & 2 & 1 & 2 & $\begin{array}{l}\text { Maintaining suitable } \\
\text { storage condition, FIFO. }\end{array}$ & $\mathrm{N}$ & & & & & PRP \\
\hline & $\begin{array}{l}\text { Biological: } \\
\text { Microbial, pests and } \\
\text { insects. }\end{array}$ & $\begin{array}{l}\text { With } \\
\text { consequences }\end{array}$ & $\begin{array}{l}\text { Transport and } \\
\text { storage } \\
\text { temperature, pest } \\
\text { control, hygiene. }\end{array}$ & 1 & 4 & 4 & $\begin{array}{l}\text { FIFO, maintaining } \\
\text { suitable storage and } \\
\text { transport temperature, } \\
\text { pest controlling plan, } \\
\text { maintain personal and } \\
\text { environmental hygiene } \\
\text { during storage and } \\
\text { transport. }\end{array}$ & $\mathrm{Y}$ & $\mathrm{Y}$ & $\bar{N}$ & $\overline{\mathrm{Y}}$ & $\mathrm{Y}$ & PRP \\
\hline \multirow[t]{3}{*}{$\begin{array}{l}\text { Taking RM } \\
\text { and PM } \\
\text { from } \\
\text { storage to } \\
\text { production }\end{array}$} & $\begin{array}{l}\text { Physical: Foreign } \\
\text { objects. }\end{array}$ & $\begin{array}{l}\text { Without } \\
\text { consequences }\end{array}$ & $\begin{array}{l}\text { Improper } \\
\text { handling and } \\
\text { personal hygiene }\end{array}$ & 3 & 1 & 3 & $\begin{array}{l}\text { Maintain personal } \\
\text { hygiene, wear mask, } \\
\text { apron, gloves, head cover } \\
\text { and beard cover, } \\
\text { maintain SOP, GHP. }\end{array}$ & $\bar{Y}$ & $\mathrm{Y}$ & $\mathrm{N}$ & $\bar{Y}$ & $\mathrm{Y}$ & PRP \\
\hline & $\begin{array}{l}\text { Chemical: } \\
\text { Unidentified }\end{array}$ & & & & & & & & & & & & \\
\hline & $\begin{array}{l}\text { Biological: } \\
\text { Unidentified }\end{array}$ & & & & & & & & & & & & \\
\hline $\begin{array}{l}\text { Batch } \\
\text { preparation } \\
\text { (weighing } \\
\text { and } \\
\text { mixing) }\end{array}$ & $\begin{array}{l}\text { Physical: Foreign } \\
\text { objects, broken egg } \\
\text { shells. }\end{array}$ & $\begin{array}{l}\text { Without } \\
\text { consequences }\end{array}$ & $\begin{array}{l}\text { Improper } \\
\text { handling and } \\
\text { personal hygiene }\end{array}$ & 2 & 1 & 2 & $\begin{array}{l}\text { Provision of filter in egg } \\
\text { broker machine, visual } \\
\text { inspection, maintaining } \\
\text { personal hygiene, wear } \\
\text { mask, apron, gloves, head } \\
\text { cover and beard cover }\end{array}$ & $\bar{Y}$ & $\mathrm{Y}$ & $\mathrm{N}$ & $\mathrm{Y}$ & $\mathrm{Y}$ & PRP \\
\hline
\end{tabular}




\begin{tabular}{|c|c|c|c|c|c|c|c|c|c|c|c|c|c|}
\hline & $\begin{array}{l}\text { Chemical: Excess } \\
\text { additives, } \\
\text { detergents etc. }\end{array}$ & $\begin{array}{l}\text { Without } \\
\text { consequences }\end{array}$ & $\begin{array}{l}\text { Incorrect hygiene } \\
\text { and cleaning, } \\
\text { faulty recipe. }\end{array}$ & 1 & 1 & 1 & $\begin{array}{l}\text { Follow the recipe and } \\
\text { recheck, timely } \\
\text { calibration of measuring } \\
\text { instruments }\end{array}$ & $\mathrm{N}$ & & & & & PRP \\
\hline & $\begin{array}{l}\text { Biological: } \\
\text { Pathogenic } \\
\text { microorganism. }\end{array}$ & $\begin{array}{l}\text { With } \\
\text { consequences }\end{array}$ & $\begin{array}{l}\text { Improper } \\
\text { personal hygiene. }\end{array}$ & 1 & 4 & 4 & $\begin{array}{l}\text { Strict personal hygiene, } \\
\text { regular hand swab tests } \\
\text { by the QA, proper } \\
\text { hygiene, food safety, and } \\
\text { behavioral training, }\end{array}$ & $\mathrm{Y}$ & $\mathrm{Y}$ & $\mathrm{N}$ & $\mathrm{Y}$ & $\mathrm{Y}$ & PRP \\
\hline \multirow[t]{3}{*}{ Mixing } & $\begin{array}{l}\text { Physical: Broken } \\
\text { piece of mixing } \\
\text { machine } \\
\text { Foreign objects }\end{array}$ & $\begin{array}{l}\text { With } \\
\text { consequences } \\
\text { Without } \\
\text { consequences }\end{array}$ & $\begin{array}{l}\text { Maintenance of } \\
\text { mixing machine, } \\
\text { improper } \\
\text { personal hygiene }\end{array}$ & 3 & 1 & 3 & $\begin{array}{l}\text { Checking the status of } \\
\text { mixing machine, } \\
\text { maintain personal } \\
\text { hygiene, wear mask, } \\
\text { apron, gloves, head cover } \\
\text { and beard cover }\end{array}$ & $\mathrm{Y}$ & $\mathrm{Y}$ & $\mathrm{N}$ & $\mathrm{Y}$ & $\mathrm{Y}$ & PRP \\
\hline & $\begin{array}{l}\text { Chemical: } \\
\text { Detergents residue. }\end{array}$ & $\begin{array}{l}\text { Without } \\
\text { consequences }\end{array}$ & $\begin{array}{l}\text { Improper } \\
\text { hygiene and } \\
\text { cleaning practice }\end{array}$ & 1 & 1 & 1 & $\begin{array}{l}\text { Maintain proper hygiene } \\
\text { and cleaning practice }\end{array}$ & $\mathrm{Y}$ & $\mathrm{Y}$ & $\mathrm{N}$ & $\mathrm{Y}$ & $\mathrm{Y}$ & PRP \\
\hline & $\begin{array}{l}\text { Biological: } \\
\text { Pathogenic } \\
\text { microorganism. }\end{array}$ & $\begin{array}{l}\text { With } \\
\text { consequences }\end{array}$ & $\begin{array}{l}\text { Improper } \\
\text { personal hygiene }\end{array}$ & 1 & 4 & 4 & $\begin{array}{l}\text { Maintain strict personal } \\
\text { hygiene, wear mask, } \\
\text { apron, gloves, head cover } \\
\text { and beard cover, keep the } \\
\text { equipment properly } \\
\text { sanitized }\end{array}$ & $\mathrm{Y}$ & $\mathrm{Y}$ & $\mathrm{N}$ & $\mathrm{Y}$ & $\mathrm{Y}$ & PRP \\
\hline \multirow[t]{3}{*}{ Dosing } & $\begin{array}{l}\text { Physical: Broken } \\
\text { piece of dosing } \\
\text { nozzle }\end{array}$ & $\begin{array}{l}\text { With } \\
\text { consequences }\end{array}$ & $\begin{array}{l}\text { Maintenance of } \\
\text { dosing machine }\end{array}$ & 1 & 1 & 1 & $\begin{array}{l}\text { Checking the status of } \\
\text { dosing machine }\end{array}$ & $\mathrm{Y}$ & $\mathrm{Y}$ & $\mathrm{N}$ & $\mathrm{Y}$ & $\mathrm{Y}$ & PRP \\
\hline & $\begin{array}{l}\text { Chemical: } \\
\text { Unidentified }\end{array}$ & & & & & & & & & & & & \\
\hline & $\begin{array}{l}\text { Biological: } \\
\text { Unidentified }\end{array}$ & & & & & & & & & & & & \\
\hline \multirow[t]{3}{*}{ Baking } & $\begin{array}{l}\text { Physical: } \\
\text { Unidentified }\end{array}$ & & & & & & & & & & & & \\
\hline & $\begin{array}{l}\text { Chemical: Moisture } \\
\text { content and water } \\
\text { activity }\end{array}$ & $\begin{array}{l}\text { Without } \\
\text { consequences }\end{array}$ & $\begin{array}{l}\text { Improper baking } \\
\text { time and } \\
\text { temperature }\end{array}$ & 3 & 1 & 3 & $\begin{array}{l}\text { Frequent lab test for } \mathrm{MC} \\
\text { and } \mathrm{a}_{\mathrm{w}} \text { from each batch, } \\
\text { maintenance of baking } \\
\text { temperature \& time as per } \\
\text { SOP }\end{array}$ & $\mathrm{N}$ & & & & & PRP \\
\hline & $\begin{array}{l}\text { Biological: } \\
\text { Microbial } \\
\text { contamination }\end{array}$ & $\begin{array}{l}\text { With } \\
\text { consequences }\end{array}$ & $\begin{array}{l}\text { Improper baking } \\
\text { time and } \\
\text { temperature }\end{array}$ & 1 & 4 & 4 & $\begin{array}{l}\text { Maintenance of baking } \\
\text { temperature \& time as per } \\
\text { SOP }\end{array}$ & $\mathrm{Y}$ & $\mathrm{Y}$ & $\mathrm{N}$ & $\mathrm{Y}$ & $\mathrm{N}$ & oPRP \\
\hline \multirow[t]{3}{*}{ Cooling } & $\begin{array}{l}\text { Physical: } \\
\text { Unidentified }\end{array}$ & & & & & & & & & & & & \\
\hline & $\begin{array}{l}\text { Chemical: Moisture } \\
\text { content and water } \\
\text { activity }\end{array}$ & $\begin{array}{l}\text { Without } \\
\text { consequences }\end{array}$ & $\begin{array}{l}\text { Improper cooling } \\
\text { time and } \\
\text { temperature }\end{array}$ & 2 & 1 & 2 & $\begin{array}{l}\text { Check for cooling } \\
\text { conveyor speed and } \\
\text { cooling temperature }\end{array}$ & $\mathrm{N}$ & & & & & PRP \\
\hline & $\begin{array}{l}\text { Biological: } \\
\text { Unidentified }\end{array}$ & & & & & & & & & & & & \\
\hline Cream & Physical: broken & With & Maintenance of & 1 & 2 & 2 & Checking the status of & $\mathrm{Y}$ & $\mathrm{Y}$ & $\mathrm{N}$ & $\mathrm{Y}$ & $\mathrm{Y}$ & PRP \\
\hline
\end{tabular}




\begin{tabular}{|c|c|c|c|c|c|c|c|c|c|c|c|c|c|}
\hline \multirow[t]{3}{*}{ filling } & $\begin{array}{l}\text { part of cream } \\
\text { dosing nozzle }\end{array}$ & consequences & $\begin{array}{l}\text { cream filling } \\
\text { machine }\end{array}$ & & & & cream filling machine & & & & & & \\
\hline & $\begin{array}{l}\text { Chemical: } \\
\text { Unidentified }\end{array}$ & & & & & & & & & & & & \\
\hline & $\begin{array}{l}\text { Biological: } \\
\text { Unidentified }\end{array}$ & & & & & & & & & & & & \\
\hline \multirow[t]{3}{*}{$\begin{array}{l}\text { Metal } \\
\text { detection }\end{array}$} & $\begin{array}{l}\text { Physical: Metal } \\
\text { parts }\end{array}$ & $\begin{array}{l}\text { With } \\
\text { consequences }\end{array}$ & $\begin{array}{l}\text { Inactive sensor } \\
\text { of the metal } \\
\text { detection } \\
\text { machine, } \\
\text { machine broken }\end{array}$ & 3 & 4 & 12 & $\begin{array}{l}\text { Frequent checking of the } \\
\text { machine status }\end{array}$ & $\mathrm{Y}$ & $\mathrm{Y}$ & $\mathrm{Y}$ & $\mathrm{N}$ & $\mathrm{N}$ & CCP \\
\hline & $\begin{array}{l}\text { Chemical: } \\
\text { Unidentified }\end{array}$ & & & & & & & & & & & & \\
\hline & $\begin{array}{l}\text { Biological: } \\
\text { Unidentified }\end{array}$ & & & & & & & & & & & & \\
\hline \multirow[t]{3}{*}{ Packaging } & $\begin{array}{l}\text { Physical: Foreign } \\
\text { parts from } \\
\text { packaging machine } \\
\text { surface, damaged } \\
\text { pack, leakage in } \\
\text { packing }\end{array}$ & $\begin{array}{l}\text { With } \\
\text { consequences }\end{array}$ & $\begin{array}{l}\text { Improper } \\
\text { cleaning, } \\
\text { shortage of } \\
\text { nitrogen gas, } \\
\text { PLC fault }\end{array}$ & 3 & 1 & 3 & $\begin{array}{l}\text { Proper cleaning of } \\
\text { packaging machine, } \\
\text { check for nitrogen gas } \\
\text { level, regular checking of } \\
\text { the PLC system }\end{array}$ & $\mathrm{Y}$ & $\mathrm{Y}$ & $\mathrm{N}$ & $\mathrm{Y}$ & $\mathrm{Y}$ & PRP \\
\hline & $\begin{array}{l}\text { Chemical: } \\
\text { Detergents residue. }\end{array}$ & $\begin{array}{l}\text { Without } \\
\text { consequences }\end{array}$ & $\begin{array}{l}\text { Improper } \\
\text { hygiene and } \\
\text { cleaning practice }\end{array}$ & 1 & 1 & 1 & $\begin{array}{l}\text { Maintain proper hygiene } \\
\text { and cleaning practice }\end{array}$ & $\mathrm{Y}$ & $\mathrm{Y}$ & $\mathrm{N}$ & $\mathrm{Y}$ & $\mathrm{Y}$ & PRP \\
\hline & $\begin{array}{l}\text { Biological: } \\
\text { Microorganisms }\end{array}$ & $\begin{array}{l}\text { With } \\
\text { consequences }\end{array}$ & $\begin{array}{l}\text { Improper } \\
\text { personal hygiene, } \\
\text { contamination in } \\
\text { packing materials }\end{array}$ & 2 & 4 & 8 & $\begin{array}{l}\text { Regular hand and surface } \\
\text { swab test, ATP check } \\
\text { before packing, strict } \\
\text { maintaining of personal } \\
\text { hygiene, QA online check } \\
\text { for leak package }\end{array}$ & $\mathrm{Y}$ & $\mathrm{Y}$ & $\mathrm{N}$ & $\mathrm{Y}$ & $\mathrm{N}$ & oPRP \\
\hline $\begin{array}{l}\text { Filling in } \\
\text { box \& date } \\
\text { coding }\end{array}$ & No hazard & $\begin{array}{l}\text { Without } \\
\text { consequences }\end{array}$ & & & & & & & & & & & $\begin{array}{l}\text { No } \\
\text { Risk }\end{array}$ \\
\hline \multirow[t]{3}{*}{$\begin{array}{l}\text { Finished } \\
\text { Goods } \\
\text { store }\end{array}$} & $\begin{array}{l}\text { Physical: Tertiary } \\
\text { package damage by } \\
\text { rodents }\end{array}$ & $\begin{array}{l}\text { Without } \\
\text { consequences }\end{array}$ & $\begin{array}{l}\text { No pest traps and } \\
\text { pest control }\end{array}$ & 2 & 1 & 2 & $\begin{array}{l}\text { Put glue trap in proper } \\
\text { places, conduct timely } \\
\text { pest control action }\end{array}$ & $\mathrm{Y}$ & $\mathrm{Y}$ & $\mathrm{N}$ & $\mathrm{Y}$ & $\mathrm{Y}$ & PRP \\
\hline & $\begin{array}{l}\text { Chemical: } \\
\text { Unidentified }\end{array}$ & & & & & & & & & & & & \\
\hline & $\begin{array}{l}\text { Biological: } \\
\text { Unidentified }\end{array}$ & & & & & & & & & & & & \\
\hline $\begin{array}{l}\text { Delivery/ } \\
\text { Transport } \\
\text { to market }\end{array}$ & No hazard & & & & & & & & & & & & No risk \\
\hline
\end{tabular}

\subsubsection{Establishing the oPRP and HACCP plan}

The HACCP plan was established for each oPRP and CCP by specifying the hazards, control measures, CL, corrective action, verification, and records management. The potential control 
points for the hazards appeared in both the raw material and the manufacturing process. Table 6 and Table 7 show the oPRP and HACCP plan in the cake manufacturing process. The HACCP control chart (Table 7) showed all the potential critical hazards that can occur during the processing steps. Gandhi, (2009) included hazard description, critical limit, observation procedure, responsible person, monitoring procedure, and corrective action in his HACCP control chart for soy milk production, whereas Burson, (2015) reported processing steps, records, and verification procedures in his meat product control chart. The HACCP control chart in the current study is similar to some previous studies (Allata et al., 2017; Pombo Marques et al., 2012; Zhao 2003). One CCP, metal detection, and two oPRP, baking and packaging, were discovered during the cake manufacturing process. The center temperature of the bakery product crumb needs to be above $100^{\circ} \mathrm{C}$ for few minutes. During baking all vegetative pathogenic bacteria should be destroyed. (Smith et al., 2002; Bryan et al. 1997). The main ingredients of cake dough include fats and oils, eggs, flour, and sugar. Among these, sugar is comparatively safe in regard to any foodborne illness. Fats and oils can be a potential source of Listeria (Wu et al., 2017). Flour, on the other hand, can pose both chemical and microbial risks. However, the most dangerous and common thing that can happen is mycotoxin, such as Aflatoxin. Despite the fact that the physicochemical properties of flour (i.e. low $\mathrm{a}_{\mathrm{w}}$ ) do not allow bacteria to grow, microorganisms can survive for extended periods of time during storage (Wu et al., 2017). As for the egg shells, they are considered as a major source of Salmonella Enteritidis (FDA, 2009b; Howard, 2012). However, cake dough is not considered a high-risk food because it has a low aw and requires baking prior to packaging and consumption (Wu et al., 2017; Clark, 2011).

Table 6. oPRPs in the cake manufacturing process

\begin{tabular}{|c|c|c|c|c|c|c|c|c|c|c|}
\hline \multirow{2}{*}{$\begin{array}{c}\text { Processing } \\
\text { Step }\end{array}$} & \multirow[t]{2}{*}{ Hazard } & \multirow{2}{*}{$\begin{array}{c}\text { Control } \\
\text { Measure(s) }\end{array}$} & \multirow[t]{2}{*}{ Critical Limit } & \multicolumn{4}{|c|}{ Monitoring procedures } & \multirow[t]{2}{*}{ Records } & \multirow{2}{*}{$\begin{array}{c}\text { Corrective } \\
\text { Action }\end{array}$} & \multirow[t]{2}{*}{ Verification } \\
\hline & & & & What? & How? & Who? & $\begin{array}{c}\text { Frequency } \\
\text { (When?) }\end{array}$ & & & \\
\hline Baking & $\begin{array}{l}\text { Biological: } \\
\text { Microbial } \\
\text { contamination }\end{array}$ & $\begin{array}{l}\text { Maintenance } \\
\text { of baking } \\
\text { temperature } \\
\& \text { time as } \\
\text { per SOP }\end{array}$ & $\begin{array}{l}\text { Baking } \\
\text { temperature } \\
\text { between } 150- \\
250^{\circ} \mathrm{C} \text { for } 5-50 \\
\text { minutes. }\end{array}$ & $\begin{array}{l}\text { Oven } \\
\text { temperature }\end{array}$ & $\begin{array}{l}\text { Physical } \\
\text { inspection }\end{array}$ & $\begin{array}{l}\text { QA officer, } \\
\text { production } \\
\text { officer }\end{array}$ & $\begin{array}{l}\text { Every one } \\
\text { hour } \\
\text { interval }\end{array}$ & $\begin{array}{l}\text { QA and } \\
\text { production } \\
\text { (Plan } \\
\text { oPRP) }\end{array}$ & $\begin{array}{l}\text { Reset the } \\
\text { temperature } \\
\text { and time, } \\
\text { reject or } \\
\text { reuse the } \\
\text { product }\end{array}$ & $\begin{array}{l}\text { Physical } \\
\text { observation } \\
\text {, calibration }\end{array}$ \\
\hline
\end{tabular}




\begin{tabular}{|c|c|c|c|c|c|c|c|c|c|c|}
\hline Packaging & $\begin{array}{l}\text { Biological: } \\
\text { Microbial } \\
\text { contamination }\end{array}$ & $\begin{array}{l}\text { Regular hand } \\
\text { and surface } \\
\text { swab test, } \\
\text { ATP check } \\
\text { before } \\
\text { packing, } \\
\text { strict } \\
\text { maintaining } \\
\text { of personal } \\
\text { hygiene, QA } \\
\text { online check } \\
\text { for leak } \\
\text { package }\end{array}$ & $\begin{array}{l}\text { Surface ATP = } \\
<50 \text { RLU; } \\
\text { Hand swab for } \\
\text { coliform = Nil; } \\
\text { Hand swab for } \\
\text { mold }=<50 \\
\text { cfu } / \mathrm{ml}\end{array}$ & $\begin{array}{l}\text { Machine } \\
\text { surface, } \\
\text { workers } \\
\text { hands }\end{array}$ & $\begin{array}{l}\text { Swab test, } \\
\text { microbiol } \\
\text { ogical test } \\
\text { in the QA } \\
\text { lab }\end{array}$ & $\begin{array}{l}\text { QA } \\
\text { Executive }\end{array}$ & $\begin{array}{l}\text { ATP test } \\
\text { every day, } \\
\text { hand swab } \\
\text { test twice } \\
\text { in a week }\end{array}$ & $\begin{array}{l}\text { QA and } \\
\text { production } \\
\text { (Plan } \\
\text { oPRP) }\end{array}$ & $\begin{array}{l}\text { Reclean the } \\
\text { surface, } \\
\text { observation } \\
\text { (hygiene } \\
\text { status) of } \\
\text { infected } \\
\text { hands } \\
\text { (person) for } \\
\text { a month, } \\
\text { reject the } \\
\text { leak pack } \\
\text { and } \\
\text { products }\end{array}$ & Recheck \\
\hline
\end{tabular}

The physical hazard of metal in cake is the most serious, and it is harmful to the consumer. Metal detectors can now be used to detect and remove metallic components during food processing. The presence of metallic components can significantly reduce food safety, resulting in perforation of human gastrointestinal tissues and surgical resection. The presence of metallic particles can cause damage to various valuable machine parts, resulting in a sudden production line shutdown and economic loss. Metal detectors are installed by food processing industrialists for product quality and safety, equipment protection, consumer satisfaction, and regulatory body requirements, among other things. Metal detectors are extremely important and effective for metal hazard control in solid seasoning powder and liquid sauces through the implementation of a HACCP plan (Chen et al., 2020). In the current study, there was no additional step in the manufacturing process that could detect or eliminate this problem, according to the process flowchart.

Table 7. HACCP Control Chart

\begin{tabular}{|c|c|c|c|c|c|c|c|c|c|c|}
\hline \multirow{2}{*}{$\begin{array}{c}\text { Processing } \\
\text { Step }\end{array}$} & \multirow[t]{2}{*}{ Hazard } & \multirow{2}{*}{$\begin{array}{c}\text { Control } \\
\text { Measure(s) }\end{array}$} & \multirow{2}{*}{$\begin{array}{l}\text { Critical } \\
\text { Limit }\end{array}$} & \multicolumn{4}{|c|}{ Monitoring procedures } & \multirow[t]{2}{*}{ Records } & \multirow{2}{*}{$\begin{array}{c}\text { Corrective } \\
\text { Action }\end{array}$} & \multirow[t]{2}{*}{ Verification } \\
\hline & & & & What? & How? & Who? & $\begin{array}{c}\text { Frequency } \\
\text { (When?) }\end{array}$ & & & \\
\hline $\begin{array}{c}\text { Metal } \\
\text { Detection }\end{array}$ & $\begin{array}{l}\text { Physical: } \\
\text { Metallic } \\
\text { component }\end{array}$ & $\begin{array}{c}\text { Detection } \\
\text { of metallic } \\
\text { component } \\
\text { by metal } \\
\text { detector }\end{array}$ & $\begin{array}{l}\text { Absence of } \\
\text { metallic } \\
\text { component }\end{array}$ & $\begin{array}{l}\text { Sensor of } \\
\text { the metal } \\
\text { detector, } \\
\text { PLC, } \\
\text { alarm. }\end{array}$ & $\begin{array}{l}\text { Physical } \\
\text { Inspection } \\
\text { and check } \\
\text { for alarm } \\
\text { (by } \\
\text { passing a } \\
\text { metal } \\
\text { under the } \\
\text { metal } \\
\text { detector) }\end{array}$ & $\begin{array}{l}\text { Maintenance } \\
\text { dept, QA \& } \\
\text { production } \\
\text { dept. }\end{array}$ & $\begin{array}{l}\text { Every } 1 \mathrm{hr} \\
\text { interval } \\
\text { during the } \\
\text { production } \\
\text { time }\end{array}$ & $\begin{array}{c}\text { QA, } \\
\text { production, } \\
\text { and } \\
\text { maintenance } \\
\text { register } \\
\text { (Plan } \\
\text { HACCP) }\end{array}$ & $\begin{array}{l}\text { Rejection of } \\
\text { the whole lot } \\
\text { of non- } \\
\text { conformed } \\
\text { products }\end{array}$ & $\begin{array}{l}\text { In house } \\
\text { checking by } \\
\text { metal pieces, } \\
\text { maintenance } \\
\text { records, } \\
\text { calibration of } \\
\text { metal detector, } \\
\text { and auditing }\end{array}$ \\
\hline
\end{tabular}




\section{Conclusion}

The HACCP system provides food manufacturers with effective preventive methods for ensuring food safety and improving the quality. Furthermore, the documentation and records generated by the HACCP system can easily assist in tracing the source of contamination, preventing further production of substandard products and reducing the consumption of manpower, material, and financial resources. The current study developed a HACCP plan for a cake manufacturing plant in Bangladesh in order to improve product safety and quality. The hazards in cake manufacturing are primarily due to the use of improper processing conditions, an unsanitary manufacturing environment, and a lack of legislative implementation. We found a CCP and two oPRPs in the entire cake manufacturing process. Further linking of the HACCP system introduced in the factory for quality management systems, such as International Organization for Standardization regulation, can potentially provide higher quality/hygiene standards, as well as increased customer awareness. However, rather than establishing the HACCP system, management teams of food corporations should ensure the application of the HACCP system in their entire production system.

\section{Acknowledgement}

We gratefully acknowledge the management team of this baking (cake) industry for their assistance throughout the research period.

\section{Declaration of interests}

There are no conflicts of interest.

\section{References}

Allata, S., Valero, A. and Benhadja, L., 2017. Implementation of traceability and food safety systems (HACCP) under the ISO 22000: 2005 standard in North Africa: The case study of an ice cream company in Algeria. Food Control, 79, 239-253. https://doi.org/10.1016/j.foodcont.2017.04.002

BAPA., 2021. Bake tech. $8^{\text {th }}$ BAPA Foodpro International Expo, 2021. Available at: https://foodpro.com.bd/baketech.php (Accessed on 1 July, 2021) 
Batista, P., Noronha, J., Oliveira, J. and Saraiva, J., 2003. HACCP generic models. Forvisao consultoria em formacao integrada. Available from: http://www.esac.pt/noronha/manuais/manual 6.pdf (in Portuguese)

Bryan, F.F., Guzewich, J.J. and Todd, E.C.D., 1997. Surveillance of foodborne disease III. Summary and presentation of data on vehicles and contributory factors; their value and limitations. J. Food Prot., 60 (6), 701-714.

Burson, D.E., 2015. Hazard Analysis Critical Control Point (HACCP) Model for Frankfurters. University of Nebraska, Lincoln, NE 68583-0908.

CAC., 1997. Hazard analysis and critical control point (HACCP) system and guidelines for its application. Annex to CAC/RCP, 3, 1-1969.

Centers for Disease Control and Prevention. 2020. List of selected multistate foodborne outbreak investigations. Available at: http://www.cdc.gov/foodsafety/outbreaks/multistate-outbreaks/outbreaks-list.html (Accessed on: May 24, 2021)

Chen, H., Liou, B. K., Dai, F. J., Chuang, P. T. and Chen, C. S., 2020. Study on the risks of metal detection in food solid seasoning powder and liquid sauce to meet the core concepts of ISO 22000:2018 based on the Taiwanese experience. Food Control, 111(145), 107071. https://doi.org/10.1016/j.foodcont.2019.107071

Clark, J.P., 2011. Implementing HACCP Plans in Bakeries. Food Technology, 65(4), 80-84.

Conforti, F. D., 2006. Cake manufacture. In Y. H. Hui (Ed.), Bakery products: Science and Technology. Ames: Blackwell Publishing. 22, 393-410.

CSPI., 2015. Outbreak Alert! 2015: A Review of Foodborne Illness in the U.S. from 20042013. Center for Science in the Public Interest. (Accessed on 03 July 2021)

Fernández-Segovia, I., Pérez-Llácer, A., Peidro, B. and Fuentes, A., 2014. Implementation of a food safety management system according to ISO 22000 in the food supplement industry: A case study. Food Control, 43, 28-34. https://doi.org/10.1016/j.foodcont.2014.02.042 
Gandhi, A.P., 2009. Development of HACCP protocols for the production of soy milk. Asian Journal of Food and Agro-Industry, 2(3), 262-279.

FAO \& WHO., 2003. Recommended international codex of practice. General principles of food hygiene, CAC/RCP 1 - 1969, Rev. 4-2003, including "Annex on Hazard Analysis Critical Control Point (HACCP) System and Guidelines for its Application".

FDA., 2009b. Oversight of egg safety. Retrieved July 11, 2016, from: www.fda.gov/newsevents/testimony/ucm115053.html

Foley, S.L., Johnson, T.J., Ricke, S.C., Nayak, R. and Danzeisen, J., 2013. Salmonella pathogenicity and host adaptation in chicken-associated serovars. Microbiology and Molecular Biology Reviews, 77(4), 582-607. https://doi.org/10.1128/MMBR.0001513

Howard, Z.R., O'Bryan, C.A., Crandall, P.G. and Ricke, S.C., 2012. Salmonella Enteritidis in shell eggs: current issues and prospects for control. Food Research International, 45(2), 755-764. https://doi.org/10.1016/j.foodres.2011.04.030

ISO 22000., 2005. Food safety management systems - requirements for any organization in the food chain, International organization for standardization.

Jones, D.R., Anderson, K.E. and Guard, J.Y., 2012. Prevalence of coliforms, Salmonella, Listeria, and Campylobacter associated with eggs and the environment of conventional cage and free-range egg production. Poultry Science, 91(5), 1195-1202. https://doi.org/10.3382/ps.2011-01795

Jubayer, M.F., Kayshar, M.S., Al Emran, M., Uddin, M.N. and Soeb, M.J.A., 2020. Response to coronavirus disease 2019: Case study of one baking industry in Dhaka, Bangladesh. Journal of Agriculture and Food Research, 2, 100077. https://doi.org/10.1016/j.jafr.2020.100077

Konstantas, A., Stamford, L., and Azapagic, A., 2019. Evaluating the environmental sustainability of cakes. Sustainable Production and Consumption. 19, 169-180. https://doi.org/10.1016/j.spc.2019.04.001 
Liu, Y., Lu, Y., Wang, L., Chang, F. and Yang, L., 2015. Survey of 11 mycotoxins in wheat flour in Hebei province, China. Food Additives \& Contaminants: Part B, 8(4), 250254. https://doi.org/10.1080/19393210.2015.1074291

Matsakidou, A., Blekas, G. and Paraskevopoulou, A., 2010. Aroma and physical characteristics of cakes prepared by replacing margarine with extra virgin olive oil. LWT-Food Science and Technology, 43(6), 949-957. https://doi.org/10.1016/j.lwt.2010.02.002

Mordor Intellegence., 2021. Cakes Market - Growth, Trends, COVID-19 Impact, and Forecasts (2021 - 2026). Available at: https://www.mordorintelligence.com/industryreports/cakes-market (Accessed on 14 May, 2021)

Pombo Marques, N.R., de Oliveira Matias, J.C., dos Reis Baptista Teixeira, R. and Ribeiro Proenca Brojo, F.M., 2012. Implementation of hazard analysis critical control points (HACCP) in a SME: case study of a bakery. Polish Journal of Food and Nutrition Sciences, 62(4), 215-227. https://doi.org/10.2478/v10222-012-0057-5

Riba, A., Bouras, N., Mokrane, S., Mathieu, F., Lebrihi, A. and Sabaou, N., 2010. Aspergillus section Flavi and aflatoxins in Algerian wheat and derived products. Food $\begin{array}{lll}\text { and } \quad \text { Chemical } & \text { Toxicology. } & \text { 2772-2777. }\end{array}$ https://doi.org/10.1016/j.fct.2010.07.005

Ricke, S. C., Jones D. R. and Gast R. K., 2013b. Eggs and egg products. In: Compendium of Methods for the Microbiological Examinations of Foods, 5th Edition (S. Doores, Y. Salfinger, \& M. L. Tortorello Eds.), 1-11, 864 Washington, D.C.: American Public Health Association.

Shuvo, S.D., Josy, M.S.K., Parvin, R., Zahid, M.A., Paul, D.K. and Elahi, M.T., 2019. Development of a HACCP-based approach to control risk factors associated with biscuit manufacturing plant, Bangladesh. Nutrition \& Food Science, 49(6), 11801194. https://doi.org/10.1108/NFS-03-2019-0074

Smith, J.P., Daifas, D.P., El-Khoury, W. and Austin, J.W., 2002. Microbial safety of bakery products. In Microbial Safety of Minimally Processed Foods, 3-34. Boca Raton: CRC Press. https://doi.org/10.4315/0362-028X-60.6.701 
Suman, S., Manyam, S., Satyanarayana, K.V. and Vijayaraghavan, K., 2021. Food safety system in Bangladesh: current status of food safety, scientific capability, and industry preparedness. Feed the future innovation lab for food safety (FSIL), USA. https://ag.purdue.edu/food-safety-innovation-lab/wp-content/uploads/2021/01/FSILFood-Safety-System-in-Bangladesh.pdf (Accessed on 5 July, 2021)

Walker, E., Pritchard, C. and Forsythe, S., 2003. Hazard analysis critical control point and prerequisite programme implementation in small and medium size food businesses. Food Control, 14(3), 169-174. https://doi.org/10.1016/S0956-7135(02)00061-0

Wallace, C. and Williams, T., 2001. Pre-requisites: a help or a hindrance to HACCP?. Food control, 12(4), pp.235-240. https://doi.org/10.1016/S0956-7135(00)00042-6

Wu, S., Ricke, S.C., Schneider, K.R. and Ahn, S., 2017. Food safety hazards associated with ready-to-bake cookie dough and its ingredients. Food control, 73, part B, 986-993. https://doi.org/10.1016/j.foodcont.2016.10.010

Zhao, M., 2003. The design of HACCP plan for a small-scale cheese plant. The Graduate school University of Wisconsin-Stout:Undergraduate. Available at: https://minds.wisconsin.edu/bitstream/handle/1793/41145/2003zhaom.pdf?sequence= 1 